\title{
Reação no estado sólido na amorfização da liga amorfa $\mathrm{Co}_{66} \mathrm{Nb}_{22} \mathrm{~B}_{15}$ Reaction in solid state amorphization in the alloy amorphous $\mathrm{Co}_{66} \mathrm{Nb}_{22} \mathrm{~B}_{15}$
}

\author{
Luciano Nascimento ${ }^{1}$
}

\begin{abstract}
Resumo
$\overline{\text { As misturas elementares de pós } \mathrm{Co}, \mathrm{Nb} \text { e } \mathrm{B} \text { com uma composição estequiométrica de 1:1:1, respectivamente }}$ preparados a partir do processamento mecânico num moinho de alta energia. A evolução da estrutura dos pós de partida e as misturas moídas foram caracterizadas por Difração de Raios X e Calorimetria Diferencial de Varredura (DSC) e Microscopia Eletrônica de Varredura e Espectroscopia por Dispersão de Energia (MEV/EDS). A liga amorfa Co-Nb-B previamente ativadas por moagem de bolas de alta energia, envolve duas reações consecutivas amorfização. A moagem mecânica, primeiramente leva a reação de amorfização entre camadas de Co e B com uma força de condução termodinâmica forte, bem como para a cinética rápida de amorfização de Co-B comparada com $\mathrm{Co}-\mathrm{Nb}$ amorfizados em fases intermetálicas. $\mathrm{Na}$ moagem ainda mais os requisitos cinéticos para uma reação de amorfização de $\mathrm{Co}-\mathrm{Nb}$ é cumprida e, consequentemente, e o surgimento de um composto $\mathrm{Nb}-\mathrm{B}$ em forma de fase amorfa. As duas fases amorfas resultantes homogeneíza-se por longo de tempo de moagem.
\end{abstract}

Palavras-chave: Moagem Mecânica. Liga Amorfa Co-Nb-B. Amorfização.

\begin{abstract}
Elemental mixtures of $\mathrm{Co}, \mathrm{Nb}$ and $\mathrm{B}$ powders of a stoichiometric composition of 1:1:1 respectively is prepared from the mechanical processing in a high energy. The structure of the starting powders and grinded mixtures were characterized by X-Ray Diffraction and Differential Scanning Calorimetry (DSC) and Scanning Electron Microscopy and Spectroscopy Dispersion Energy (SEM / EDS). The amorphous alloy, $\mathrm{Co}-\mathrm{Nb}-\mathrm{B}$ was previously activated by high energy milling balls, involves two consecutive reactions amorphization. The mechanical milling, first leads to amorphization reaction between layers of $\mathrm{Co}$ and $\mathrm{B}$ with a strong thermodynamic driving force as well as for the rapid kinetics $\mathrm{Co}-\mathrm{B}$ amorphization compared with $\mathrm{Co}-\mathrm{Nb}$ intermetallic phases in amorphization. In further grinding the kinetic requirements for amorphization of $\mathrm{Co}-\mathrm{Nb}$ reaction is observed and, consequently, the appearance and $\mathrm{Nb}-\mathrm{B}$ compound in the form of amorphous phases. The two amorphous phases resulting homogenized by grinding over time.
\end{abstract}

Keywords: Mechanical Alloying. Amorphous Alloy Co-Nb-B. Amorphization.

\footnotetext{
${ }^{1}$ Doutor em Engenharia Química, Programa de Pós-Graduação em Engenharia Química, Centro de Tecnologia e Geociências-CTG/ UFPE. luciano.ufpe@gmail.com
} 


\section{Introdução}

Amorfização de estado sólido a partir de seus análogos de fases cristalinas tem sido intensamente estudada nas últimas décadas (JOHNSON, 1986).

A moagem de alta energia ou moagem mecânica é uma ferramenta de processo de não equilíbrio que permite a elaboração de materiais estáveis e / ou metaestáveis, tais como solução sólida supersaturada, ligas amorfas, nanocompósitos e compostos intermetálicos para armazenamento de hidrogênio e para reações catalíticas (NASCIMENTO, 2013). Amorfização por moagem mecânica tm sido sugerido para ser semelhante ao de amorfização de estado sólido em estruturas em camadas alternadas e superestruturadas usando ligas nanocristalinas.

Geralmente, muitas composições de ligas nanocristalinas (amorfas e quasicristalinas), sofrem um aumento em suas propriedades mecânicas através da cristalização parcial controlada na sua estrutura amorfa, que forma uma microestrutura constituída de matriz amorfa e de cristais de Al- $\alpha$ homogeneamente as dispersas na matriz em grãos com tamanhos nanométricos (FADEEVA; LENOV, 1996). Entretanto a baixa estabilidade térmica destes materiais amorfos é devido à fácil cristalização erecristalização A reação de amorfização do estado sólido que incluem: irradiação, amorfização assistida por hidrogênio, interdifusão de metais elementares, vitrificação por pressão induzida e deformação mecânica, sendo muito importante em processos catalíticos.

A formação da fase amorfa é conhecida por ser criticamente dependente das condições iniciais de moagem, totalmente dependente do tempo. É importante ressaltar a formação de uma fase intermetálica cristalina acontece antes da amorfização no estado sólido, por se tratar de reações peritéticas em solução sólida. Segundo BONETTI et al., (1990) descreveu que em muitos sistemas o processo de moagem em moinho de alta energia com pós elementares, formam uma sequência de fases formadas com o tempo em solução sólida saturada, intermetálica e amorfa. Se numa fase intermetálica se forma numa fase de solução sólida (reação de estado sólido), antes de uma fase amorfa via processo de amorfização, que dependente diretamente das energias livres relativas, ou seja, em função do tempo de moagem (JANG; KOCH, 1990). Para FAN et al., (1997) o processo de amorfização em ligas amorfas seque a seguinte sequência : fase ordenada $\rightarrow$ fase desordenada (perda de ordem de longo alcance) $\rightarrow$ fase de granulação fina (nanocristalina) $\rightarrow$ fase amorfa. Neste sistema, estas observações aconselha que a energia de moagem é totalmente crescente, implicando numa mudança de estado físico que libera calor num processo exotérmico, com maior liberação de calor, maior temperatura, que pode cristalizar a fase amorfa ou mesmo recristalizar. Os materiais amorfos são metaestáveis, e quando submetidos a aquecimento cristalizam em estruturas mais estáveis formando fases cristalinas. De maneira geral essa cristalização não ocorre num único evento, mas numa faixa de temperaturas em diversos eventos. Em certas composições estequiométricas, as denominadas de ligas nanocristalinas, as suas propriedades mecânicas podem ser ainda mais altas, devido aos processos de cristalização parcial controlada na estrutura amorfa, que forma uma microestrutura de grãos refinado, na matriz amorfa e de pequenos cristais em formato de cristalitos na fase eutética. O ganho de energia livre resultante da mistura dos elementos é sugerido para ser a força motriz para a amorfização, por processo de sondagem a frio. Neste último caso, no entanto, quando a energia livre do composto intermetálico é levantada por deformação mecânica que frequente acima do homólogo amorfo, pode ocorrer uma transformação de fase polimorfa de cristalização vítrea rica em fases de óxidos mistos. A máxima faixa de amorfização é observada em valores de intensidade baixa de moagem que não fornece um aenergia suficiente para amorfização, enquanto 
intensidades elevadas cristalizam a fase amorfa formada. Os defeitos também aumentam a energia livre do sistema do intermetálico a um nível mais alto do que na fase amorfa e consequentemente fica possível a formação da fase amorfa.

\section{MURTY e RANGANATHAN}

decreveram que o processo de amorfização acontece quando há uma forte tensão na sua difusão lenta, pois tem sido demonstrado que os materiais amorfos podem também ser obtidos por desestabilizar a cristalinidade de uma fase sólida por reação em estado sólido alcançando assim o seu máximo de fases desejáveis. As ligas Co-Nb-B (Vidros Metálicos de Grandes Volumes (BMGs) possuem excelentes propriedades físicas e químicas, as quais são bastante promissoras para várias aplicações) possui uma ligação covalente junto com elemento metalóide (B) e alto módulo elasticidade com o metal de transição (Co) (AZZAZA; ALLEG; SUÑOL, 2013). Além disso, o $\mathrm{Nb}$ e $\mathrm{B}$ têm entalpia negativa de mistura com o elemento constituinte majoritário Co, é possível ajustar as propriedades mecânicas dos vidros metálicos de grandes volumes da liga amorfa Co-Nb-B através do controle da interação atômica de aglomerados na matriz vítrea (DUN; LIU; SHEN, 2012). O Boro é um elemento geralmente utilizado em composições amorfas e nanocristalinas como um antigo elemento de vidro e para estender a estabilidade térmica destes materiais metaestáveis. A presença de $\mathrm{Nb}$, na matriz da liga amorfa causa inibição do crescimento de grãos resultam numa estrutura nanocristalina. O objetivo deste trabalho é preparar, caracterizar e estudar o mecanismo de estabilidade da amorfização por transformação de fase cinética que ocorre com o aumento de temperatura de cristalização térmica devida o tempo de moagem de 5 horas, possiblitando as reações de estado sólido em na liga amorfa $\mathrm{Co}_{66} \mathrm{Nb}_{22} \mathrm{~B}_{15}$.

\section{Materiais e Métodos}

Os pós dos elementos cobalto, nióbio e boro, conforme a sua granulometria (peneira de 100 mesh, tendo uma pureza de $99,9 \%$ cada um dos elementos, provenientes da Aldrich Chemical, foram pesados nas proporções adequadas à composição $\left(\mathrm{Co}_{66} \mathrm{Nb}_{22} \mathrm{~B}_{15}\right)$ e homogeneizados mecanicamente. Os componentes foram pesados numa balança de precisão, modelo: Micronal B4000 com resolução de $10^{-2} \mathrm{~g}$ obtendo-se as composições nominais.

A relação bola/pó determinada foi de 20:1, indicando assim o peso total da amostra, $25 \mathrm{~g}$, como também o peso das esferas de aço cromo, com três tamanhos diferentes (6 esferas de $20 \mathrm{~mm}, 4$ esferas de $15 \mathrm{~mm}$ e 6 esferas de $10 \mathrm{~mm}$ ), perfazendo um total de $225 \mathrm{~g}$ de esferas. Este material foi colocado em uma jarra de aço de alta dureza, a qual foi selada para obtenção de vácuo de $5 \times 10^{-2}$ mbar, prevenindo possível contaminação dos pós.

A jarra com os a mistura dos pós-elementares foi então colocada num moinho planetário de Modelo: NQM2L Mill Pulverizer onde foi moído inicialmente por $5 \mathrm{~h}$, com rotação de 300 rpm. Para a identificação das fases da liga a amorfa $\left(\mathrm{Co}_{66} \mathrm{Nb}_{22} \mathrm{~B}_{15}\right)$, foi usado um difratômetro Shimadzu XRD 6000, utilizando radiação de CuK $\alpha$ com comprimento de onda de 1,5406 ̊. As medidas foram tomadas para uma ampla gama de ângulos de difração (20) que variam de $20^{\circ}$ a $120^{\circ}$ com passo angular de $0,05^{\circ}$ e com tempo de contagem por ponto igual a $4 \mathrm{~s}$.

A análise em MEV/EDS foi realizada com um microscópio eletrônico varredura SHIMADZU SUPERSCAN SSX-550 com uma tensão de aceleração de 0,5 a $30 \mathrm{kV}$ com uma etapa de $10 \mathrm{~V}$, após a amostra ter sido revestido com uma fina camada de ouro depositada em vácuo, a fim de melhorar o contraste. A análise química foi realizada através por Espectroscopia de Energia Dispersiva (EDS). A estabilidade térmica foi avaliada por meio de calorimetria de diferencial varredura (DSC Netzsch 404) a uma velocidade de aquecimento de $0,67 \mathrm{~K} / \mathrm{s}$ sob uma atmosfera de fluxo de ar. 


\section{Resultados e Discussão}

\section{Difração de raios $X$}

Observando-se a Figura 1, sem tratamento térmico, verifica-se que não aparecem picos característicos de fases cristalinas, sendo possível notar apenas um halo de difração, evidenciando o estado amorfo nesta amostra e confirmando o aspecto vítreo da mesma. O Aparecimento de picos simples de forma bem expandida são características das estruturas amorfas. O que comprova o êxito na amorfização da liga estudada com estequiometria $\mathrm{Co}_{66} \mathrm{Nb}_{22} \mathrm{~B}_{15}$. A fase inicial amorfa é nula começando, a se processa a partir da mistura de fases puras entre os pós de elementos $\mathrm{Co}, \mathrm{Nb}$ e de $\mathrm{B}$ cristalinos; porém, após a uma forte coesão dos elementos da liga, surgindo subsequencialmente um forte incremento de curva com fases desconhecidas. Estas fases são óxido intermetálicos que passa a dominar a reação em estado sólido com uma alta metaestabilidade de cristalização.
A presença de pequenos picos é correspondente à formação de uma fase amorfa e cristalina da liga muito rica em óxido intermetálico; entretanto a pouca intensidade indica que a fração volumétrica desta fase não é representativa e pode ser ao processo de fases amorfas intermetálica formado no segundo pico de cristalização, corresponde a nanocristalização do Cobalto e Nióbio amortizado com o Boro na rede.

Observou-se que alguns picos não identificados e pequena intensidade é relativamente às fases amorfas intermetálicas metaestáveis $\varphi-\left(\mathrm{Co}_{2} \mathrm{Nb}_{8} \mathrm{~B}_{2}\right.$, $\mathrm{Co}_{1} \mathrm{Nb}_{3} \mathrm{~B}_{5}, \mathrm{Co}_{2} \mathrm{Nb}, \mathrm{Nb}_{2} \mathrm{~B}_{3}$ ) são compostas cristalinas e intermetálicas em solução sólida, coexistindo com a fase amorfa. O efeito visto no difratograma mostra um forte alargamento de pico, sendo um fenômeno comum no processamento da liga amorfa devido à presença de fases intermetálicas e amorfas em conjuntas que coexistem, formando uma única fase.

Figura 1. Espectro de difração de raios $X$ apresentando fases na liga amorfa $\mathrm{Co}_{66} \mathrm{Nb}_{22} \mathrm{~B}_{15}$.

$$
\varphi-\left(\mathrm{CO}_{2} \mathrm{Nb}_{8} \mathrm{~B}_{2}, \mathrm{CO}_{1} \mathrm{Nb}_{3} \mathrm{~B}_{5}, \mathrm{CO}_{2} \mathrm{Nb}, \mathrm{Nb}_{2} \mathrm{~B}_{3}\right)
$$

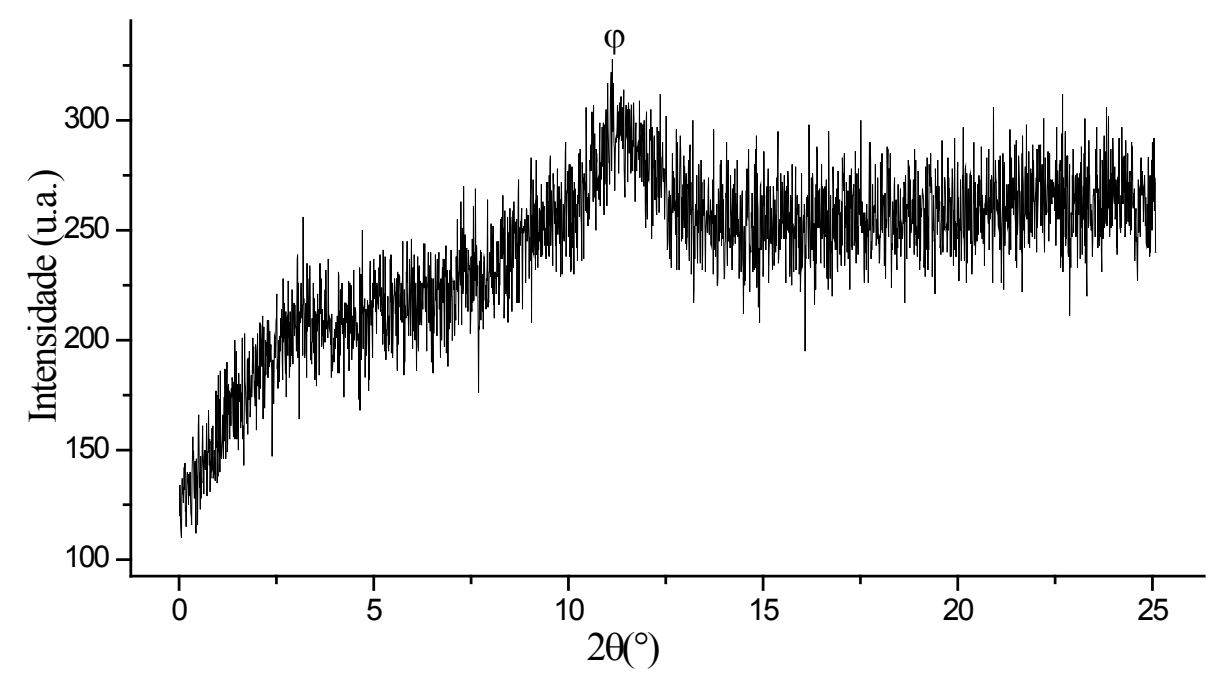

Fonte: Autor 
Microscopia eletrônica de varredura e EDS

A Figura 2 mostra o pó na liga amorfa $\mathrm{Co}_{66} \mathrm{Nb}_{22} \mathrm{~B}_{15}$, o resultado da amostra de Microscopia Eletrônica de Varredura na amostra revela pequenos cristais de simetrias irregulares com grãos deformados e sendo muito oxidado com Co e $\mathrm{Nb}$. Os aglomerados de partículas irregulares com bordas e pequenos poros, onde se podem observar pequenos flocos amorfos. Esta aglomeração de partículas é um fenômeno comum resultante das forças de Van der Waals. Além disso, as fraturas grosseiras das partículas finas e soldadas a frio ocorrem simultaneamente coma diminuição da fragilidade das colisões entre as paredes da jarra e o choque entre as bolas devido um aumento de sua temperatura e da energia cinética. Isto levou a formação de partículas menores com formas irregulares e bem aglomeradas com tamanho de $5 \mu \mathrm{m}$. O constante crescimento da camada de óxidos tem sido determinado pelas as fases existente entre o Cobalto e o Nióbio em determinada temperatura de fusão de $1200^{\circ} \mathrm{C}$ na formação da liga, tendo assim constantes metafases entre as fases amorfas e intermetálicas presente no grão com formatos irregulares e aglomerados de formatos poucos esféricos com nódulos em toda a sua superfície.

Figura 2. $\mathrm{MEV}$ da liga amorfa $\mathrm{Co}_{66} \mathrm{Nb}_{22} \mathrm{~B}_{15}$
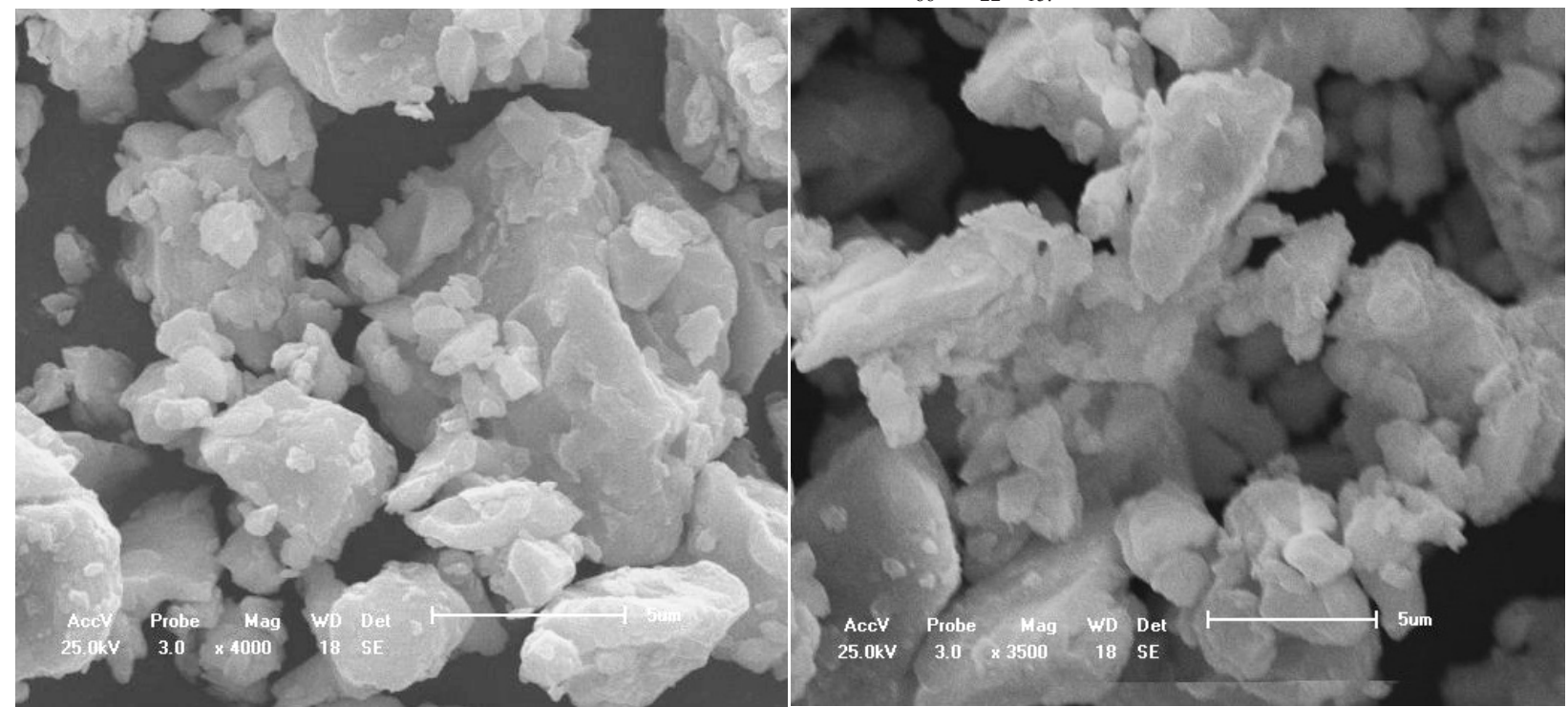

Fonte: Autor

A Espectroscopia Dispersiva de Energia (EDS) utilizada na análise da liga amorfa $\mathrm{Co}_{66} \mathrm{Nb}_{22} \mathrm{~B}_{15}$ com seus respectivos espectro coberto com fina camada de ouro, fora descritos na Figura 3, onde exibe os elementos de maior concentração para o cobalto e o nióbio com a presença pequena quantidades de oxigênio que possibilita a formação de óxidos em sua superfície. O oxigênio forma óxido com o cobalto e nióbio que possibilita a reação peritética entre a as fases intermetálicas da liga amorfa, formando uma fina camada de óxido intermetálico. 
Figura 3. EDS do pós da liga amorfa $\mathrm{Co}_{66} \mathrm{Nb}_{22} \mathrm{~B}_{15}$ obtido por moagem de alta energia.

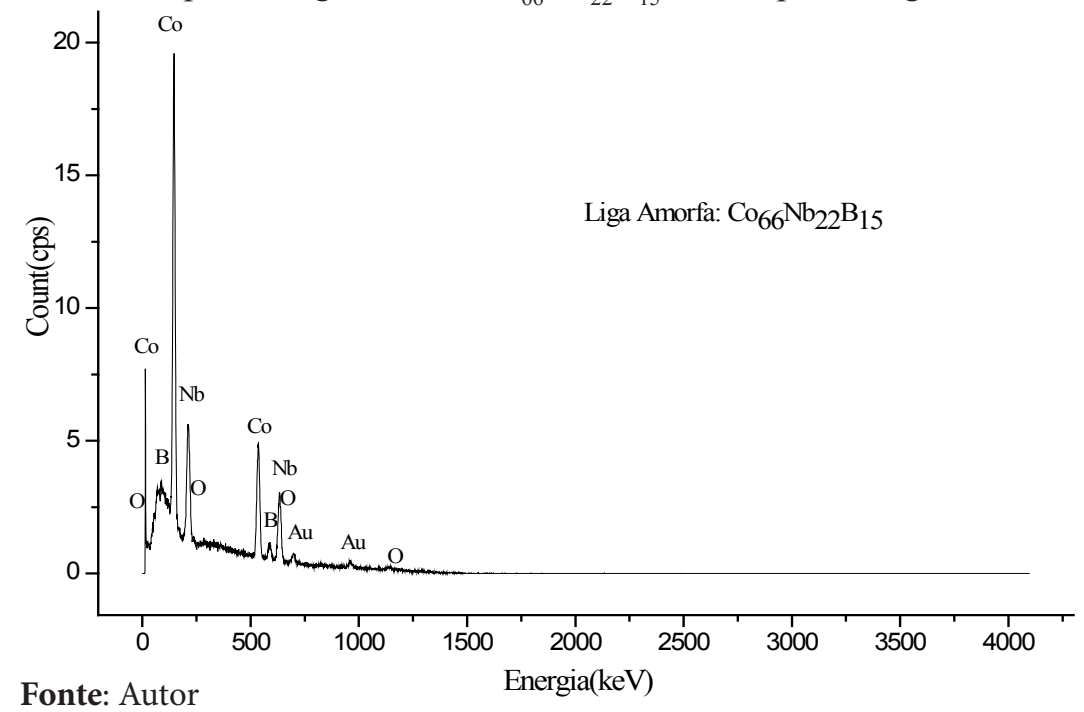

\section{Calorimetria Diferencial de Varredura}

Uma curva típica de DSC da liga amorfa $\mathrm{Co}_{66} \mathrm{Nb}_{22} \mathrm{~B}_{15}$ utilizado neste trabalho está mostrado na Figura 4. O termograma de DSC foi obtido a uma taxa de aquecimento constante de $10^{\circ} \mathrm{C} /$ min, que depende da velocidade de aquecimento, numa faixa de $557-750^{\circ} \mathrm{C}$. A curva é típica de uma estrutura amorfa, que apresenta um pico endotérmico correspondente ao evento de transição do vidro metálico amorfo em um líquido superresfriado seguido de um pico exotérmico. Podemos observar que, a transformação estrutural na fase final ocorre em etapas diferentes com uma diferente concentração de boro na estrutura amorfa.

A temperatura de início de transição vítrea $\left(\mathrm{T}_{\mathrm{g}}\right)$ em torno de $649^{\circ} \mathrm{C}$ e o evento de cristalização $\left(\mathrm{T}_{\mathrm{x}}\right)$ em $675^{\circ} \mathrm{C}$, pois os resultados experimentais indicam que o $\Delta \mathrm{T}_{\mathrm{x}}$ foi melhorado com o aumento do conteúdo de Co, atingindo o valor mais elevado na composição $\mathrm{Co}_{66} \mathrm{Nb}_{22} \mathrm{~B}_{12}$, e para um maior aumento em conteúdo de $\mathrm{Nb}$ há uma diminuição de $\Delta \mathrm{T}_{\mathrm{x}}$, quando a estrutura amorfiza devido o Boro fazer uma solução supersólida na liga amorfa.

Figura 4. Termograma de DSC obtido a uma taxa de aquecimento constante de $10^{\circ} \mathrm{C} / \mathrm{min}$

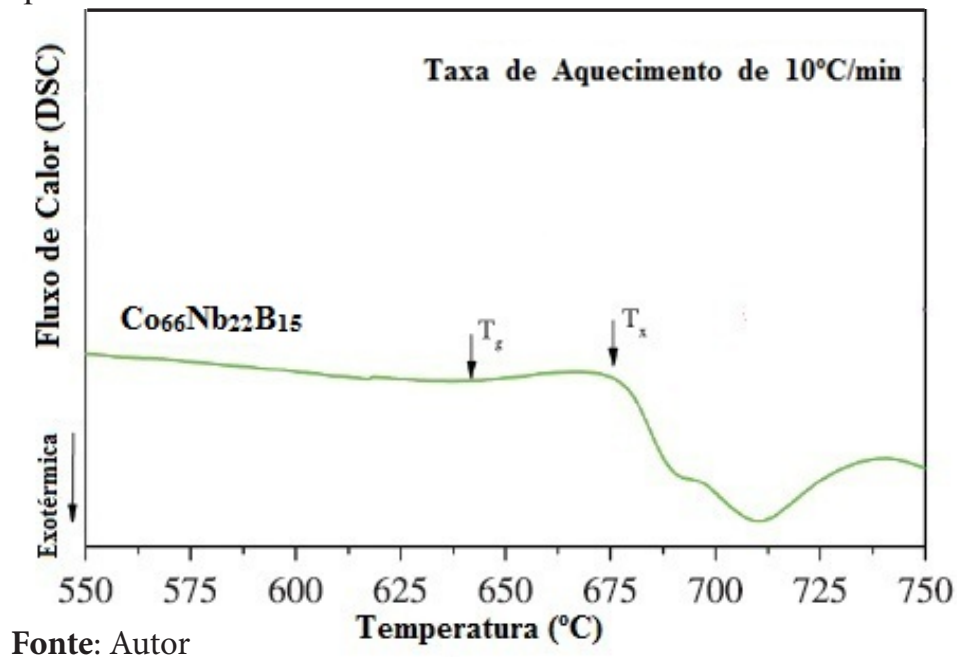




\section{Conclusão}

- Através do Difratograma de Raios x, observa um alagamento bem suave; curva típica das estruturas amorfas, isto também é confirmado pela evolução das proporções de fase como uma função do tempo de moagem em $5 \mathrm{~h}$;

- Análise do MEV mostra as morfologias das partículas de pó durante uma moagem de 5 horas revelando estruturas com formatos irregulares e plaquetas As alterações na morfologia durante o processo de moagem são devido à competição entre a fratura, a soldadura a frio, a aglomeração e deaglomeração das partículas de pó, sendo a amorfizados devido ao Boro que é adicionado entre a matriz de $\mathrm{Co}-\mathrm{Nb}$;

- O resultado mostra um aumento para as temperaturas de $\mathrm{T}_{\mathrm{g}}$ e $\Delta \mathrm{T}_{\mathrm{x}}$ na composição perto do ponto eutético, provando assim um maior aumento na formação da amorfização no estado sólido para outras formações de fases amorfas /intermetálica;

- Dependendo da mistura inicial, mudança estrutural dos pós mecanicamente moído ocorre a seguinte forma: refinamento de grão, solução sólida de difusão e / ou a formação de novas fases amorfas, onde podemos ver no EDS maiores concentrações de $\mathrm{Co}$ e $\mathrm{Nb}$, se complexando com Oxigênio e com o Boro;

- $\mathrm{Na}$ moagem mecânica, a formação de complexos de boretos complexado com o Co e $\mathrm{Nb}$ são sujeitas a defeitos contínuos que levam a uma alteração gradual na energia livre, tendo entalpias negativas na liga. Assim, a mistura de pó é completamente transformando numa estrutura amorfa após $5 \mathrm{~h}$ de moagem o que é confirmado pelo aparecimento de a temperatura de transição vítrea de cerca $675^{\circ} \mathrm{C}$, aumentando o estado de amorfização.

\section{Agradecimentos}

Os autores agradecem ao PRH 28 / MCT / ANP pelo o apoio financeiro deste trabalho e ao Departamento de Física e a Central Analítica do Departamento de Química Fundamental do Centro de Ciências Exatas e da Natureza da UFPE.

\section{Referências}

AZZAZA, S.; ALleG, S.; SUÑOL, J. J. Phase Transformation in the ball milled Fe $31 \mathrm{Co}_{31} \mathrm{Nb}_{8} \mathrm{~B}_{30}$ Powders. Advances in Materials Physics and Chemistry, n. 3, p. 90-100, 2013.

BONETTI, E.; COCCO, G., ENZO, S.; VALDRÈ, G. Amorphization and phase transformations of mechanically alloyed Ti-Al powders: electron microscopy investigation. Material Science and Technology, n. 6, p.1258-1262, 1990.

DUN, C.; LIU, H.; SHEN, B. Enhancement of plasticity in $\mathrm{Co}-\mathrm{Nb}-\mathrm{B}$ ternary bulk metallic glasses with ultrahigh strength. Journal of NonCrystalline Solids, n. 358, p. 3060-3064, 2012.

FADEEVA, V. I.; LENOV, A. V. Amorphization and Crystallization of Al-Fe by Mechanical Alloying. Mater. Sci. Eng. A, n. 206, p. 90-94, 1996.

FAN, G. J.; GUO, F. Q.; HU, Z. Q; QUAN, M. $\mathrm{X}$.; LU, K. Amorphization of selenium induced by high-energy ball milling. Physical Review B, v. 55, n.17, p. 11011-11013, 1997.

JANG, J. S. C. KOCH, C. C. Amorphization and Disordering of the Ni-Al Ordered Intermetallic by Mechanical Milling. J. Mater. Res,. n. 5, p. 498-510, 1990.

JOHNSON, W. L. Thermodynamic and kinetic aspects of the crystal to glass transformation in metallic materials. Progress in Materials Science, n. 30, p. 81-134, 1986.

MURTY, B. S., RANGANATHAN, S. Novel materials synthesis by mechanical alloying/milling. Int. Mater. Rev., v. 43, n. 3, p. 101-41, 1998. 
NASCIMENTO, L. Estudo das ligas amorfas na reação de oxidação com aplicabilidade na síntese do metanol e produção de olefinas. 2013. Projeto de Tese (Doutorado em Engenharia Química) Universidade Federal de Pernambuco, Recife-PE. 